\title{
Latent Heat Saturation in Microstructural Evolution by Severe Plastic Deformation
}

\author{
Mattia Bacca $^{\mathrm{a}, \mathrm{b},{ }^{*}}$ and Robert M. McMeeking ${ }^{\mathrm{a}, \mathrm{b}, \mathrm{c}, \mathrm{d}}$ \\ ${ }^{a}$ Mechanical Engineering Department, University of California, Santa Barbara CA 93106, USA \\ ${ }^{\mathrm{b}}$ Materials Department, University of California, Santa Barbara CA 93106, USA

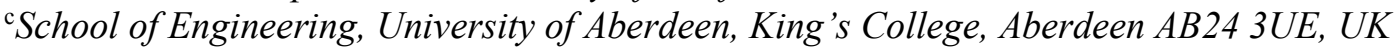 \\ dINM - Leibniz Institute for New Materials, Campus D2 2, 66123 Saarbrücken, Germany
}

\begin{abstract}
During plastic deformation, most of the dissipated work is released as heat, but a fraction of it, usually small, is stored in the microstructure, is called latent heat and is associated with the network of dislocations that develops. The rate of energy storage in the microstructure divided by the rate of plastic dissipation is defined as the latent heat capacity. Latent heat remains stored in the microstructure of cold worked specimens after quenching. This energy is associated with modified mechanical properties, e.g. hardness, and is released upon annealing. Saturation of this stored energy has been observed in experiments after a specific amount of plastic deformation is reached. A thermodynamically consistent model for continuous dynamic recrystallization is proposed in this paper with the aim of explaining the phenomenon of latent heat saturation and relating it to grain refinement. The proposed model has three essential features: (i) the latent heat increases in the specimen during plastic deformation as plastic work is continuously dissipated; (ii) the rate of latent heat storage per unit work, i.e. the latent heat capacity, is related to the internal architecture of the microstructure and decreases to zero as a consequence of microstructural evolution; (iii) the relationship between the latent heat and the microstructure is described through the use of two parameters: (a) the dislocation density and (b) the average grain diameter. A comparison of the proposed model with experiments is reported and a validation for the prediction of microstructural evolution, as well as the evolution of the latent heat and latent heat capacity, is provided.
\end{abstract}

Keywords: Microstructural evolution; Latent heat; Reverse Hall-Petch effect; Recrystallization.

\section{Introduction}

The mechanical properties of a material depend on its physical state, and in particular, for solid materials, on the configuration of the microstructure. Many authors have developed constitutive models that are sensitive to the heterogeneity of the material, as well as its microstructure, for

\footnotetext{
${ }^{*}$ Corresponding author.

E-mail address: mattia.bacca@engineering.ucsb.edu.
} 
small deformations (Mindlin 1964, Bacca et al. 2013) and for large deformations (Aifantis 1984). Less attention has been dedicated to the development of constitutive models that are sensitive to the evolution of the microstructure, as well as the mechanical properties linked to it. The proposed work attempts to contribute to this field on a thermodynamically consistent basis.

Microstructural evolution occurs via mechanical processes that involve cold working of the material. The resulting change in microstructure is associated with the amount of plastic deformation experienced by the material and becomes significant after severe plastic deformation (SPD) associated with processes such as low-speed machining (Shankar et al. 2005, Bacca, Hayhurst and McMeeking 2015). Experiments performed by Taylor and Quinney (1934) on torsion of metal rods have shown how this evolution is measured by latent heat, i.e. the energy that remains stored in the specimen after the specimen is quenched. This energy is considered to be stored in the microstructure, is responsible for the evolution of the microstructure as it is associated with the network of dislocations that develops, and increases continuously with plastic work (Bever, Holt and Titchener 1973). The Taylor and Quinney (1934) experiments on torsion, as well as experiments on machining (Saldana, King and Chandrasekar 2012) demonstrate saturation of the latent heat for a finite amount of plastic strain, with continuous reduction to zero of the rate of storage of latent heat per unit work (called the latent heat capacity).

Some constitutive models based on latent heat storage have previously been developed with the aim of predicting microstructure evolution in metals (Bernacki et al. 2009, Le and Kochmann 2009, Quan et al. 2011, Brown and Bammann 2012, Bacca et al. 2015), with a focus on the phenomenon of dynamic recrystallization. This phenomenon has also been studied with different approaches such as phase field models (Militzer 2011, Moelans et al. 2013, Sreekala and Haataja 2007, Suwa, Saito and Onodera 2008), which considers the nucleation and subsequent growth of new grains within the "old" microstructure, and with the use of finite element techniques as well as other methodologies such as cellular automata (Hallberg 2011). However, the latent heat saturation (LHS) phenomenon is neglected in those models and a physical interpretation has not been reported in the literature.

In the present paper, a thermodynamically consistent model for continuous dynamic recrystallization (CDRX) created by SPD is provided. In this model, an evolution of the micro and nanomechanisms that accommodate macroscopic plastic deformation is considered, and a physical interpretation for the phenomenon of LHS is provided.

As will be explained in this paper, grain refinement is created by CDRX and gives a reduction of the average grain diameter, allowing the microstructure ultimately to develop a grain boundary sliding (GBS) mechanism for plastic deformation. When this mechanism of plastic deformation becomes predominant over other mechanisms associated with dislocation motion, microstructure evolution is complete and no further energy can be stored in the system as latent heat, giving LHS.

An explanation of the proposed model and all its features is provided in Section 2; results on the prediction of the evolution of the microstructure as well as the latent heat and latent heat capacity 
are given in Section 3; discussion of the model and its conditions of applicability are reported in Section 4; finally some conclusions are drawn in Section 5.

\section{The constitutive model}

\subsection{Basic principles}

In the present work, an improved version of the simple model for CDRX developed by Bacca et al. (2015) is developed. The basic principles of the original model are explained in this section, with more detail given in Bacca et al. (2015), while the proposed new features are explained in the subsequent sections.

In the model the yield strength is given by superposition of two main contributions: Dislocation strengthening, which mechanism involves pinning of dislocations by others, as described by Taylor (1934); (ii) Grain boundary strengthening, as described by Hall (1951) and Petch (1953). The total yield strength in shear, in agreement with Castro-Fernandez and Sellars (1989) and Le and Kochmann (2009), has the form

$$
\tau_{y}=\alpha b \mu \sqrt{\rho}+\lambda\left(\frac{1}{\sqrt{d}}-\frac{1}{\sqrt{d_{0}}}\right)
$$

where $\alpha$ is a phenomenological constant for Taylor hardening (usually taken as unity), $b$ is the Burgers vector, $\mu$ is the shear modulus, $\rho$ is the dislocation density, $\lambda$ is the Hall-Petch coefficient, $d$ is the current average grain size, and $d_{0}$ is a reference grain size that is sufficiently large so that the Hall-Petch effect is negligible. With condition $d=d_{0}$, eq. (1) only accounts for changes to hardness caused by changes to $\rho$.

During plastic deformation, energy conservation can be written neglecting reversibly stored macroscopic elastic strain energy, i.e. identifying only dissipative terms, in order to obtain the rate equation (with superposed dots indicating differentiation with respect to time or rates)

$$
\tau_{y} \dot{\gamma}_{p}=\dot{Q}+\dot{U}_{m}
$$

where $\dot{\gamma}_{p}$ is the rate of change of the shear equivalent plastic strain, $\dot{Q}$ is the rate per unit volume at which mechanical work is dissipated as heat, and $\dot{U}_{m}$ is the rate per unit volume at which plastic work is stored in the microstructure as latent heat (the latent heat rate) (Taylor and Quinney 1934). The right-hand side of eq. (2) corresponds to the amount of plastic work per unit volume supplied to the system at the unit time and can be associated with the rate of change of a hardening energy potential $W_{p}$, for which $\tau_{y}=\partial W_{p} / \partial \gamma_{p}$, thus $\dot{W}_{p}=\tau_{y} \dot{\gamma}_{p}$ (Chang and Kochmann 2015). The left-hand side of eq. (2) is the rate per unit volume at which the applied loads do plastic work on the material. If we define a latent heat capacity, $\kappa$, as the rate at which plastic work is stored in the microstructure as latent heat, divided by the rate of total plastic work, we have 
$\dot{U}_{m}=\kappa \tau_{y} \dot{\gamma}_{p}$

As will be explained in section $2.3 \kappa$ tends to reduce for continued plastic strain.

Experimental measurement of energy storage and dissipation have been carried out mostly through observations of the Taylor and Quinney (T\&Q) coefficient, $\beta$, which determines the rate at which plastic work is dissipated as heat divided by the rate of total plastic work, giving

$\dot{Q}=\beta \tau_{y} \dot{\gamma}_{p}$

Eq. (3) and eq. (4) can be substituted into eq. (2) to give

$\kappa=1-\beta$

The microstructural energy $U_{m}$, also known as the latent heat, is assumed to be as described by Berdichevsky (2006), which is given by the sum of two contributions due to dislocation density, $\rho$, and grain size, $d$, respectively, such that

$U_{m}(\rho, \mathrm{d})=-k \mu\left[\log \left(1-\sqrt{\frac{\rho}{\rho_{s}}}\right)+\sqrt{\frac{\rho}{\rho_{s}}}\right]+\frac{\chi \Gamma_{s}}{2 d}$,

where $k$ is a constant, $\chi$ is a grain shape factor (its value is 3 if we consider spherical grains), $\Gamma_{s}$ is the average interface energy of the grain boundaries, and $\rho_{s}$ is a theoretical saturation limit for the dislocation density, i.e. in the absence of recrystallization $\rho_{s}$ is the maximum dislocation storage capacity, for which the latent heat becomes indefinitely large (Berdichevsky 2006). The constant $k$ is defined by $k \mu=\kappa_{0} \tau_{s} \gamma_{0}$ (Bacca et al. 2015), where $\kappa_{0}$ is the initial latent heat capacity (associated with zero plastic strain), and $\tau_{s}$ and $\gamma_{0}$ are parameters that will be defined later in this section.

Differentiation of eq. (6) and substitution of the result into eq. (3), gives

$$
\frac{k \mu \dot{\rho}}{2 \rho_{s}\left(1-\sqrt{\rho / \rho_{s}}\right)}-\frac{\chi \Gamma_{s}}{2 d^{2}} \dot{d}=\kappa \tau_{y} \dot{\gamma}_{p}
$$

which is a differential equation that relates the microstructural parameters $\rho$ and $d$ to the shear equivalent plastic strain $\gamma_{p}$.

As many theories and experiments indicate, CDRX commences only after a critical amount of plastic deformation is reached, associated with instability in the intragranular dislocation structure (Bailey and Hirsch 1962, Luton and Sellars 1969, Roberts and Ahlblom 1978, Quan et al. 2011). This instability leads to the creation of low angle subgrain boundaries condensed from 
existing dislocations in the microstructure. At this point, CDRX has started and all existing dislocations and many of the new ones produced will eventually be adsorbed into the subgrain boundaries, causing increasing misorientation across them until new, distinct, refined grains are created.

From the above observations, microstructural evolution is divided into two main stages (Le and Kochmann 2009, Bacca et al. 2015): prior to recrystallization, and during recrystallization. The first stage is summarized by the conditions $\dot{d}=0, d=d_{0}$, as no grain refinement occurs at this stage, and $\kappa=\kappa_{0}$, which implies that $\kappa$ can evolve only during CDRX.

These conditions allow us to integrate eq. (7) and obtain a relationship between $\gamma_{p}$ and $\rho$. From this, a relationship between $\gamma_{p}$ and $\tau_{y}$ is obtained by using eq. (1) and has the form of the Voce law (Voce 1948)

$$
\tau_{y}=\tau_{0}+\left(\tau_{s}-\tau_{0}\right)\left[1-\exp \left(-\gamma_{p} / \gamma_{0}\right)\right]
$$

valid for $\gamma_{p} \leq \gamma_{c}$, where $\gamma_{c}$ is the critical amount of plastic strain for initiation of CDRX. The parameter $\tau_{0}$ is the initial yield strength in shear, $\tau_{s}$ introduced above in connection with eq. (6) is given by $\tau_{s}=\alpha b \mu \sqrt{\rho_{s}}$, is the theoretical maximum value of $\tau_{y}$ that can be reached if no recrystallization occurs and is associated with the maximum dislocation density $\rho_{s}$ that can be stored by the microstructure, and $\gamma_{0}$ is a representative shear strain, also introduced above. The parameters $\gamma_{0}$ and $\tau_{s}$ are influenced by strain rate and temperature as measured by Kocks (1976). We take implicit account of this influence, as the material parameters used in the model are calibrated to the evolution of hardness for continued plastic strain at constant strain rate and temperature. The dislocation density $\rho$ in this stage is obtained from eq. (1), giving $\rho_{0}=\left(\tau_{0} / \alpha b \mu\right)^{2}$ as the initial dislocation density. The relationship between yield strength and plastic strain included in eq. (8) is empirical as it arises from experimental observations. It has been used by Berdichevsky (2006) to formulate the first term on the right hand side of eq. (6) so it unsurprisingly generates eq. (8) when grain size is constrained to be $d_{0}$ and eq. (7) is integrated.

When CDRX starts, we adopt the condition of steady state yield strength as experiments do not evidence significant strain hardening or softening during this process (Le and Kochmann 2009). Thus

$$
\tau_{y}=\tau_{c}, \dot{\tau}_{y}=0
$$


where $\tau_{c}$ is obtained from eq. (8) for $\gamma_{p}=\gamma_{c}$, and corresponds to the critical yield strength in shear when recrystallization starts. Substituting eq. (1) into eq. (9) and the result into eq. (7), we have

$$
\left[\frac{\lambda k \sqrt{\rho(d)}}{2 \alpha b \rho_{s} d^{3 / 2}\left(1-\sqrt{\rho(d) / \rho_{s}}\right)}-\frac{\chi \Gamma_{s}}{2 d^{2}}\right] \dot{d}=\kappa \tau_{c} \dot{\gamma}_{p}
$$

with

$$
\sqrt{\rho(d)}=\frac{1}{\alpha b \mu}\left[\tau_{c}-\lambda\left(\frac{1}{\sqrt{d}}-\frac{1}{\sqrt{d_{0}}}\right)\right]
$$

The results in eq. (10) and (11), with the hypothesis of constant latent heat capacity $\kappa=\kappa_{0}$, have been integrated by Bacca et al. (2015) to obtain qualitative matching with experiments on machining. The hypothesis of $\kappa=\kappa_{0}$ gives indefinite increase of the latent heat during continued plastic strain, in disagreement with experiments, and leads to an underestimation of the grain size for large plastic deformations. The proposed, modified model, as will be explained in the next sections, considers a microstructure dependent latent heat capacity in order to overcome the aforementioned limitations and to give better agreement with experiments.

\subsection{Grain boundary sliding mechanism}

Macroscopic plastic deformation is accommodated through deformation mechanisms at the micro and nanoscale. In the proposed, modified model, two main mechanisms are identified as responsible for plastic deformation: (i) dislocation motion (mainly dislocation glide) (DM) and (ii) grain boundary sliding (GBS). The plastic strain rate is described as the superposition of the strain rate generated by each single mechanism, as proposed by Langdon (1994), giving, for the shear component, the condition

$\dot{\gamma}_{p}=\dot{\gamma}_{\mathrm{DM}}+\dot{\gamma}_{\mathrm{GBS}}$

A phenomenological formulation that describes both terms on the right-hand side of eq. (12), for high temperature creep and superplasticity, has been proposed by Langdon (1994) as

$$
\dot{\gamma}_{i}=\frac{A_{i} D_{i} \mu b}{\bar{k} T}\left(\frac{b}{d}\right)^{p_{i}}\left(\frac{\tau_{y}}{\mu}\right)^{n_{i}}, \quad i=\mathrm{DM}, \mathrm{GBS}
$$

where $\bar{k}$ is the Boltzmann constant, $T$ is the temperature, $D_{i}$ is the appropriate diffusion coefficient, $A_{i}, p_{i}$ and $n_{i}$ are positive constants calibrated from experiments. The result in eq. (13) reveals the influence of grain size $d$ on GBS activity through $b / d$, the ratio of the average size $b$ 
of the core of a gliding dislocation to the average size $d$ of a sliding grain (Rachinger 1952-53). The exponents on the grain size term in eq. (13) are deduced to be $p_{\mathrm{DM}}=0$, and $p_{\mathrm{GBS}}=1$ for most experiments (Langdon 1994). Earlier work by Raj and Ashby (1971) provided a similar model to describe GBS with $p_{\mathrm{GBS}}=2$ and proved its validity also at low temperature and small grain size. We observe negligible discrepancy in the results of our model for $p_{\mathrm{GBS}}$ that ranges from 1 to 2 ; thus we have chosen $p_{\mathrm{GBS}}=1$. Given the adopted values for $p_{\mathrm{DM}}$ and $p_{\mathrm{GBS}}$, increasing GBS activity is observed as grains become smaller while DM activity remains independent of the grain size. The latter observation suggests a limitation on the validity of eq. (13) when grain refinement from CDRX is involved; i.e. dislocation adsorption caused by recrystallization should diminish DM activity. This leads to an implicit dependence of the DM strain rate contribution on the grain size $d$, a dependence not captured by Langdon's model. To overcome this limitation, we take both $\dot{\gamma}_{\mathrm{DM}}$ and $\dot{\gamma}_{\mathrm{GBS}}$ to be grain size dependent, with only the latter one described by eq.(13), so that the fractions they contribute to the total strain rate change as the grain size diminishes, with the GBS fraction rising (according to eq. (13)) and the DM one falling.

When the grain size reaches the nanoscale, experiments show a mismatch with respect to the Hall-Petch law, as expressed in the second term on the right-hand side of eq. (1). In this regime GBS substitutes for DM as the primary deformation mechanism, and it is observed that the yield strength diminishes with a reduction in grain size (Chokshi et al. 1989). This reverse Hall-Petch effect arises because the energy barrier to GBS is less in microstructures with smaller grains as less geometric distortion is needed to accommodate the resulting plastic flow. Experiments indicate a critical grain size $d_{c}$ at which the transition between the Hall-Petch effect and its reverse occurs (Chokshi et al. 1989). This critical grain size is influenced by the temperature, for the aluminum alloy Al6061-T6 at room temperature this grain size is around $100 \mathrm{~nm}$ (Maung, Earthman and Mohamed 2012), and at that transition DM ceases. From the latter observation, and the dependence of $\dot{\gamma}$ GBS on $d$ in eq. (13), we deduce the form

$\dot{\gamma}_{\mathrm{GBS}}(d)=\dot{\gamma}_{p} \frac{d c_{c}}{d}, \dot{\gamma}_{\mathrm{DM}}(d)=\dot{\gamma}_{p}\left(1-\frac{d_{c}}{d}\right)$

where $\dot{\gamma}_{p} d_{c}$ summarizes all the constants included in eq. (13) with the exception of $d$. Thus for $d$ $=d_{c}, \dot{\gamma}_{\mathrm{GBS}}=\dot{\gamma}_{p}$ and $\dot{\gamma}_{\mathrm{DM}}=0$. We now consider $d=d_{0}$, where $d_{0}$ as above is the grain size prior to any refinement, in a situation where $d_{c}=100 \mathrm{~nm}$ and $d_{0}=75 \mu \mathrm{m}$ as in the case of experimental data for A16061-T6 (Shankar et al. 2005, Maung et al. 2012). In this situation, $\dot{\gamma}$ $\mathrm{GBS} \approx 0$, and $\dot{\gamma}_{\mathrm{DM}} \approx \dot{\gamma}_{p}$. This observation shows that DM dominates the strain rate at large grain size prior to refinement, but GBS has taken over entirely if CDRX reduces the grain size to 100 $\mathrm{nm}$, in agreement with the experimental observations.

\subsection{Saturation of the latent heat}

In eq. (4), the T\&Q coefficient $\beta$ depends on the microstructural configuration (grain size $d$ and dislocation density $\rho$ ) as the heat generation mechanism will change as a consequence of 
microstructural evolution. Superposition of the strain rates due to GBS and DM in eq. (12) is extended to heat generation in eq. (4) as

$\dot{Q}=\beta_{\mathrm{DM}} \tau_{y} \dot{\gamma}_{\mathrm{DM}}+\beta_{\mathrm{GBS}} \tau_{y} \dot{\gamma}_{\mathrm{GBS}}$,

where $\beta$ DM and $\beta$ GBS are partial T\&Q coefficients related to each individual mechanism. We take these to be constant and thus independent of $\rho$ and $d$. Furthermore, we consider $\beta$ GBS $=1$, based on the observation that GBS lacks a mechanism for increasing the energy that is globally stored in the microstructure. This is in contrast to DM where Frank-Read sources multiply dislocations and leave them stored statistically in the microstructure, including their associated elastic strain and dislocation core energies. In most nanocrystalline metals, GBS has been observed to be accompanied by grain boundary rotations (Shan et al. 2004), which can increase the boundary misorientation angle and, according to the Read and Shokley (1950) equation, the grain boundary energy $\Gamma_{s}{ }^{1}$. This occurs by adsorption of intragranular statistically stored dislocations which then become geometrically necessary to accommodate the increased misorientation of the grain boundary. In such reconfiguration, the energy stored by a single dislocation that transits from the forest to the grain boundary becomes smaller (not greater), therefore there is no global increase of energy $U_{m}$ associated with GBS.

If we substitute eq. (14) into eq. (15) to calculate $\beta$ and substitute it into eq. (5), we obtain an expression for the latent heat capacity $\kappa$ as a function of the average grain size $d$ that, with the initial condition $\kappa\left(d_{0}\right)=\kappa_{0}$, gives

$\kappa(d)=\kappa_{0}\left(\frac{1-d_{c} / d}{1-d_{c} / d_{0}}\right)$

The result in eq. (16) gives $\kappa=0$ for $d=d_{c}$ which implies saturation of the latent heat for a critical grain size. This is in agreement with the hypothesis of negligible DM and the consequent impossibility of storage of energy in the microstructure by the generation of dislocations.

\section{Results}

\subsection{Physical parameters and calibration of the model}

Figure 1 reports a schematic representation of the machining experiments of Shankar et al. (2005) used for calibration and subsequent validation of the proposed model. The parameters of the experiment are given in Table 1 , where we state the cutting speed $v_{0}$, the rake angle $\vartheta$, and the feed $a_{0}$, defined in Figure 1. In this experiment, the total shear equivalent plastic strain $\gamma_{p}$ inside the chip is related to the rake angle and has been determined with an empirical relationship through the measurement of the chip thickness. In Table 1 we report three different values of the rake angle and the total plastic strain that is associated with each value. Figure 1 also shows the primary deformation zone (PDZ) where the strain is developed and where the material transits

\footnotetext{
${ }^{1}$ However we neglect this change as a first approximation.
} 
from its annealed state (in the domain of the "material being removed") to the cold worked state (inside the chip). A relevant strain rate is observed in the PDZ while outside this domain the strain rate is negligible. In this experiment a low cutting speed is also adopted in order to sustain the hypothesis of negligible temperature rise and allow for the consideration of $T \approx T_{r}$ (with $T_{r}$ the room temperature).

\begin{tabular}{|l|c|l|l|l|}
\hline Parameter/Measurements & Symbol & Values \\
\hline Rake angle & $\vartheta$ & $20^{\circ}$ & $-5^{\circ}$ & $-20^{\circ}$ \\
\hline Cutting speed & $v_{0}$ & \multicolumn{4}{|c|}{$10 \mathrm{~mm} / \mathrm{s}$} \\
\hline Feed & $a_{0}$ & \multicolumn{3}{|c|}{$0.150 \mathrm{~mm}$} \\
\hline $\begin{array}{l}\text { Shear equivalent plastic } \\
\text { strain }\end{array}$ & $\gamma_{\mathrm{p}}$ & 1.7 & 3.2 & 5.2 \\
\hline $\begin{array}{l}\text { Average grain size after } \\
\text { machining }\end{array}$ & $d$ & $150 \mathrm{~nm}$ & $82 \mathrm{~nm}$ & $75 \mathrm{~nm}$ \\
\hline
\end{tabular}

Table 1: Parameters and measurements for the machining experiment of Shankar et al. (2005).

The experiments of Shankar et al. (2005) utilize A16061-T6 specimens. This material is an aluminum alloy, and its physical properties are given in Table 2 .

\begin{tabular}{|l|c|l|}
\hline Parameter & Symbol & Value \\
\hline Shear modulus & $\mu$ & $25 \mathrm{GPa}^{*}$ \\
\hline Grain shape factor & $\chi$ & $3^{*}$ \\
\hline Grain Interface Energy & $\Gamma_{s}$ & $0.625 \mathrm{~J} / \mathrm{m}^{2 *}$ \\
\hline Hall-Petch constant & $\lambda$ & $0.01 \mathrm{MPa} \mathrm{m^{1/2 }}$ \\
\hline Taylor constant & $\alpha$ & $1^{* *}$ \\
\hline Burger vector & $b$ & $2.86 \cdot 10^{-10} \mathrm{~m}^{* * *}$ \\
\hline Grain size when annealed & $d_{0}$ & $75 \mu \mathrm{m}^{* * * *}$ \\
\hline $\begin{array}{l}\text { Critical grain size for inversion } \\
\text { of the Hall-Petch effect }\end{array}$ & $d_{c}$ & $100 \mathrm{~nm}^{* * * * *}$ \\
\hline
\end{tabular}

Table 2: Material parameters for Al6061-T6. The sources are * Le and Kochmann 2009, ${ }^{* *}$ Taylor 1934, ${ }^{* * *}$ Rösler, Harders and Bäker (2007), ${ }^{* * * *}$ Shankar et al. 2005, ${ }^{* * * * *}$ Maung et al. 2012.

In addition to the parameters given in Table 2, the other parameters appearing in eq. (8) through (11) are reported in Table 3. These parameters are obtained from the calibration carried out by Bacca et al. (2015) by use of the results of experiments by Shankar et al. (2005). 


\begin{tabular}{|l|c|l|}
\hline Parameter & Symbol & Value \\
\hline Initial yield strength in shear & $\tau_{0}$ & $186.91 \mathrm{MPa}$ \\
\hline $\begin{array}{l}\text { Yield strength in shear for } \\
\text { steady state conditions }\end{array}$ & $\tau_{c}$ & $258.27 \mathrm{MPa}$ \\
\hline $\begin{array}{l}\text { Saturation yield strength in } \\
\text { shear }\end{array}$ & $\tau_{s}$ & $1.74 \mathrm{GPa}$ \\
\hline Initial latent heat capacity & $\kappa_{0}$ & $12 \%$ \\
\hline Reference shear strain & $\gamma_{0}$ & 1.04 \\
\hline $\begin{array}{l}\text { Critical shear equivalent plastic } \\
\text { strain }\end{array}$ & $\gamma_{c}$ & 0.005 \\
\hline
\end{tabular}

Table 3: Calibrated parameters for the model based on machining experiments for the aluminum alloy A16061-T6 (Shankar et al. 2005). For details about the calibration see Bacca et al. (2015).

As can be observed in Table 3, $\tau_{s}$ is comparable to the theoretical shear strength given by $\mu / 2 \pi$. This is due to the phenomenon of saturation of dislocations which gives a microstructure that is such densely populated by defects that the activation energy needed for creating plastic deformation is comparable to the energy needed to deform a perfect crystal.

\subsection{Theoretical results and comparison with experiments}

Figure 2 shows the mechanical behavior of Al6061-T6 subject to large plastic deformations, for the same strain rate and temperature as in the Shankar et al. (2005) machining experiments. For $\gamma_{p}<\gamma_{c}$ the behavior is described by eq. (8), whereas for $\gamma_{p}>\gamma_{c}$ the mechanical behavior is described by eq. (9) as perfectly plastic. These theoretical values are indicated with a solid line, while the experimental values (taken from Shankar et al. 2005) are indicated with open circles and relative error bars. This figure reports the evolution of the Vicker hardness $H_{v}$ for an increasing plastic strain, as this quantity is associated with the yield strength in shear by the relationship $\tau_{y}=H_{v} / 3 \sqrt{3}$ (Nurislamova et al. 2008). In this comparison, the model has been calibrated by choosing $\tau_{0}$ and $\tau_{c}$ in order to give minimum discrepancy between the associated $H_{0}$ and $H_{c}$ and all the experimental points (in the range described by the error bars). With the choice reported in Table 3, the deviation of the proposed horizontal line from the limits of the error bars of the experimental data points is on average $0.67 \%$ and has a maximum of $2.6 \%$. The error committed in the measurement of the hardness, in the same data points (taking one half of the amplitude of the error bars in Figure 2), is on average $2.13 \%$ with a maximum of $3.07 \%$. From this observation it follows that the error committed in the approximation of the hardening behavior with the proposed model is smaller than the error committed in the obtainment of the values of the data points in Figure 2. Therefore the proposed model is considered to be in good agreement with experiments.

The parameters' value reported in Tables 2-3 can be utilized for the numerical integration of eq. (10) and (11), with the substitution of $\kappa$ taken from eq. (16), in order to give the evolution of the 
grain size $d$ for continued plastic strain. This law is reported in Figure 3 in the solid line, while the dashed line represents the same integration but with $\kappa=\kappa_{0}$ as in the previous model we developed (Bacca et al. 2015) ${ }^{2}$. On the abscissa is the shear equivalent plastic strain $\gamma_{p}$ while on the ordinate is a logarithmic scale of the grain size ratio $d / d_{0}$. Given the singularity of the function $d\left(\gamma_{p}\right)$ in its first derivative for $\gamma_{p}=\gamma_{c}$, which gives $d\left(\gamma_{c}\right)=-\infty$ (Bacca et al. 2015), we chose to integrate eq. (10) and (11) in an implicit form with respect to $d$ so that we obtain the function $\gamma_{p}(d)$. This numerical integral has been calculated through a forward Euler method with a fixed step $\Delta d=-10^{-8} d_{0}$, initial value $d_{0}$, up to the final value $d_{c}$. The latter value is asymptotically reached by the solid line of Figure 3 for very large $\gamma_{p}$ as the rate of change of $d$, according to eq. (10) and (16), becomes 0 when $d=d_{c}$.

The evolution of the microstructure, in terms of grain refinement created by CDRX, has been observed experimentally by Shankar et al. (2005), and the grain size has been measured for three different amounts of plastic strain, as shown in Table 1. These results are indicated in Figure 3 with open circles and relative error bars $( \pm 50 \%)$ and compared with the curves discussed in the previous paragraph.

This figure shows that our current model gives excellent agreement with the experimental data, passing within the error bars for all the three experimental results. In addition, the figure shows how the current model, unlike the previous one (dashed line), captures the horizontal asymptote for the grain size at large plastic strains, at $d=d_{c}$.

Figure 3 also shows that a nanometric grain size such as $d_{c}$ is the smallest obtainable by grain refinement caused by CDRX driven by plastic deformation. When such grain size is reached, GBS becomes a predominant deformation mechanism, and the system ceases to store energy in the microstructure. As a consequence, the driving force for the formation of new grain boundaries is negligible. At this point, the latent heat has saturated and no further grain refinement can occur.

In Figure 4 the evolution of the latent heat capacity is shown for the current model (solid line) and compared with that of our previous model (dashed line). These results are obtained from the ones shown with the solid line in Figure 3 through the use of eq. (16), for the proposed model in the solid line. A continuous decrease to zero is visible in the graph for the current model, which starts from the initial value of 0.12 , while a constant value of 0.12 is assumed in our previous model.

Early experiments on the measurement of the latent heat were done by Taylor and coworkers. They believed the fraction of cold work being stored (as latent heat), as well as the fraction that is dissipated, was a constant material property. Later on it has been observed that these fractions are determined by the amount of plastic strain (Taylor and Quinney 1934); however, little influence has been noted in regards to strain rate (Hodowany et al. 2000). The temperature is also expected to play an important role in the energy storage, as high temperatures have the capability to release the latent heat by annealing. In the proposed model, the temperature has

${ }^{2}$ For this case analytical solution of eq. (10) and (11) can be obtained and is reported in Bacca et al. (2015). 
been considered constant and close to its ambient room value. In support of this hypothesis most of the experiments considered, and performed at room temperature, have been observed to give a modest temperature rise (Farren and Taylor 1925, Rosenhein and Stott 1933, and Kanzaki 1951, Shankar et al. 2005), such that the mechanisms of energy storage and deformation are not affected by it.

In Figure 5 we show the evolution of the ratio of the dissipated heat $Q$ to the amount of plastic work $W_{p}$ done to the specimen for an increasing plastic strain. We call this ratio: the mean T\&Q coefficient, and it is given by

$\bar{\beta}=1-\frac{U_{m}}{W_{p}}$, with $W_{p}=\int_{0}^{\gamma_{p}} \tau_{y} d \gamma_{p}$,

The curve of $\bar{\beta}$ versus the shear equivalent plastic strain $\gamma_{p}$ is shown by the solid line while the experimental results are shown by data points of various shapes. The theoretical results are obtained through substitution of eq. (11) into eq. (6) from the value of $d$ shown in Figure 3, for $U_{m}$ (see the description of Figure 7 for more detail), and by integration of eq. (8) and (9), for $W_{p}$. The experimental results are taken from Farren and Taylor (1925) (circles), Rosenhein and Stott (1933) (triangles), and Kanzaki (1951) (squares). All these authors used commercially pure aluminum specimens while the model has been calibrated to the machining experiments of Shankar et al. (2005), which uses Al6061-T6 specimens. Given the difficulties we encountered in retrieving ex perimental data on the specific alloy considered, we consider this comparison to be appropriate for a qualitative investigation as is well known that even minute amount of changes to the alloying composition result in relevant changes in the plastic behavior.

Figure 5 shows qualitative agreement between the theory and the experiments, and the experimental results are in qualitative agreement with each other. Farren and Taylor (1925) and Rosenhein and Stott (1933) performed mechanical tests in tension for a bar and a wire respectively, while Kanzaki (1951) performed compression tests on bars. These experiments were all carried out at room temperature but at different strain rates, we compare them by virtue of the negligible influence of the latter parameter on the phenomenon of energy storage (Hodowany et al. 2000).

In Figure 6 the T\&Q coefficient is shown as a function of the shear equivalent plastic strain $\gamma_{p}$ and is compared with experiments. The theoretical results are taken from Figure 4 by using eq. (5). The experimental results considered are taken from Mason, Rosakis and Ravichandran (1994) for Al 2024 specimens, and by Hodowany et al. (2000) for Al 2024-T3 specimens. Also in this case the materials are different form the one considered for the calibration of the model therefore the same consideration made for Figure 5 applies in this figure. Both sets of experimental results are obtained from high strain rate compression tests on a Kolsky bar. The comparison between the theory and the experiments should be limited to $0.85 \leq \beta \leq 1.00$ which is the range that is in agreement with the commonly accepted values from Taylor and coworkers (Farren and Taylor 1925, and Taylor and Quinney 1934). Within this range we observe a good matching for strains that are larger than 0.1 for Mason et al. (1994), and for strains larger than 
0.4 for Hodowany et al. (2000). All three curves show a tendency towards a horizontal asymptote at $\beta=1$, that corresponds to LHS.

In Figure 7 the evolution of the latent heat for continued plastic strain is shown for the current model (solid line) and compared with our previous model (dashed line). These results are obtained from the results shown in Figure 3. For each value of $\gamma_{p}$, its associated value of $d$ is substituted into eq. (6) together with the result of eq. (11) to give the corresponding value for the latent heat $U_{m}$.

As evidenced by this figure, LHS leads to a continuous decrease in the slope of the curve until a steady state value (saturation) of the latent heat is obtained. Our previous model (Bacca et al. 2015), associated with the dashed line, did not capture this asymptotic behavior leaving the latent heat to increase indefinitely for continued plastic strain. The gap between the solid line and the dashed line represents the energy dissipated into heat by the increased GBS activity caused by grain refinement.

In Figure 8 we show the evolution of the deformation mechanism in terms of the ratio of the strain rate that is accommodated by GBS to the total strain rate. These results are obtained from the results shown in Figure 3. For each value of $\gamma_{p}$, its associated value of $d$ is substituted into eq. (14).

In Figure 9, the ratio of the energy stored by the grain boundaries $U_{d}$ (the second term on the right-hand side of eq. (6)) to the total latent heat $U_{m}$ is shown as a function of plastic strain. These results are taken from the calculation made to obtain the results in Figure 7, by considering only the second term on the right-hand side of eq. (6) to obtain $U_{d}$. For an increasing plastic strain $U_{d}$ increases as a fraction of the latent heat as more grain boundary surface is developed by grain refinement. An asymptotic value of $83.34 \%$ is observed for this curve.

Stability of the nanostructure obtained by grain refinement, in metal alloys, is provided by second phase precipitates, which pins the grain boundaries inhibiting their annihilation created by grain growth. In these materials, the activation energy for grain growth can be much higher than the energy needed to activate recovery, which annihilates dislocations and releases their stored energy. As a consequence, grain refinement is especially desirable in metal alloys, as the latent heat redistribution shown in Figure 9 confers more stability to the increased hardness associated with the increase in latent heat obtained by cold working if compared to cold worked pure metals and metal alloys that have no grain refinement.

\subsection{Finite element simulation of machining}

The machining process involved in the experiments of Shankar et al. (2005) has been simulated with the finite element (FE) software Advant Edge (Third Wave Systems [http:// www.thirdwavesys.com/]). In this simulation, the mechanical behavior of the material subject to plastic deformation is described by a user yield surface (UYS) and by a yield criterion. The UYS is a numerical algorithm that determines the value of the yield strength and other state variables for a given plastic strain. We encoded the proposed model as a UYS and choose the Von Mises yield criterion. In this case the yield strength in shear is obtained from eq. (8) for $\gamma_{p} \leq \gamma_{c}$, and 
from eq. (9) for $\gamma_{p}>\gamma_{c}$ as shown in Figure 2. As state variables for the material, we chose to consider the microstructural parameter $d$ and the energy storage parameters $\kappa, \beta, U_{m}$ and $U_{d} / U_{m}$. The variable $d$ can be evaluated numerically for a given plastic strain $\gamma_{p}$ by integrating eq. (10) and (11), as reported in Section 3.2 and shown in Figure 3. With the purpose of reducing the computational time for the simulation, under the hypothesis of constant strain rate $\dot{\gamma}$ and temperature $T$, we introduce an explicit function $d\left(\gamma_{p}\right)$ that approximates the curve of Figure 3 with minimum discrepancy and provides the value of $d$ for any given value of $\gamma_{p}$

$d\left(\gamma_{p}\right)= \begin{cases}d_{0} & \gamma_{p} \leq \gamma_{c} \\ d_{0}+\left(d_{c}-d_{0}\right)\left\{1-\exp \left[-\left(\frac{\gamma_{p}-\gamma_{c}}{\gamma_{1}}\right)^{n}\right]\right\} & \gamma_{p}>\gamma_{c}\end{cases}$

where $\gamma_{1}$ and $n$ are parameters that have to be calibrated in order to minimize the discrepancy between eq. (18) and the plot in Figure 3. For the case shown in Figure 3, associated with the machining experiment of Shankar et al. (2005), these parameters have values $\gamma_{1}=1.4310^{-5}$ and $n$ $=0.2$. It should be noted that eq. (18) is only a numerical approximation of the result of the model proposed in Section 2, therefore the reader should just take it as a suggestion for an explicit formulation of the function $d\left(\gamma_{p}\right)$ that has the purpose of save computational time. Moreover, if a different experiment is considered, i.e. a different set of strain rate $\dot{\gamma}$ and temperature $T$ is adopted (holding the hypothesis of negligible temperature rise and constant strain rate), the parameters in Table 2-3 should be re-determined, eq. (10) and (11) should be integrated again with the new data and the values of the parameters $\gamma_{1}$ and $n$ should be recalculated.

In Figure 10 we show the contour plot relative to the grain size $d$ distribution for the machining simulation, by using eq. (18). The friction coefficient between the cutting tool and the chip is taken as 0.5 , the cutting speed is chosen as the minimum allowed by the software, which is 20 $\mathrm{mm} / \mathrm{s}$ (two times bigger than the cutting speed in Shankar et al. 2005, but still in the same order of magnitude), the rake angle is $20^{\circ}$, the feed a (see Figure 1) is $0.15 \mathrm{~mm}$, and the material of the specimen is the aluminum alloy Al6061-T6. Similarly, as observed by Bacca et al. (2015), relevant grain refinement takes place in the chip domain, including the primary deformation zone, as well as in a substrate that extends for $0.1 \mathrm{~mm}$ below the machined surface. In these regions, a minimum grain of $d_{c}=100 \mathrm{~nm}$ is shown in the blue contour. Outside these regions, the material has comparable properties with the same material in annealed conditions and is shown in the red contour. For the latter conditions, a grain size of $d_{0}=75 \mu \mathrm{m}$ is considered, in agreement with Shankar et al. (2005).

Figure 11 shows the contour plot relative to the distribution of the latent heat capacity $\kappa$. The value of $\kappa$ is obtained, for a given shear plastic strain $\gamma_{p}$, by substitution of $d$, taken from eq. (18), into eq. (16). A minimum value of 0 (which corresponds to LHS) is predicted within the regions of maximum grain refinement in the blue contour. The material in annealed conditions shows the maximum value of 0.12 in the red contour. 
Figure 12 shows the contour plot relative to the distribution of the T\&Q coefficient $\beta$. The value of $\beta$ is obtained by substitution of $\kappa$, at the previous paragraph, into eq. (5). A maximum value of 1 (which corresponds to LHS) is predicted within the regions of maximum grain refinement in the red contour. The material in annealed conditions shows the minimum value of 0.88 in the cyan contour.

Figure 13 shows the contour plot relative to the distribution of the latent heat $U_{m}$. The value of $U_{m}$ is obtained, for a given shear plastic strain $\gamma_{p}$, by substitution of $d$, taken from eq. (18), into eq. (11) and this, together with $d$, into eq. (6). A maximum value of $1.1410^{7} \mathrm{~J} / \mathrm{m}^{3}$ is predicted within the regions of maximum grain refinement in the red contour. The material in annealed conditions shows the minimum value of $1.3610^{6} \mathrm{~J} / \mathrm{m}^{3}$ in the blue contour. For the sake of comparison with experiments, the difference between the saturated latent heat and the latent heat in annealed conditions is considered as $\Delta U_{m}=110^{7} \mathrm{~J} / \mathrm{m}^{3}$. Taking aluminum density as $\hat{\rho}=2.7$ $10^{6} \mathrm{~g} / \mathrm{m}^{3}$, we have $\Delta U_{m}=0.88 \mathrm{cal} / \mathrm{g}$, in agreement with the observations of Kanzaki (1951), and considering aluminum molar volume $V_{m}=110^{-5} \mathrm{~m}^{3} / \mathrm{mol}$, we have $\Delta U_{m}=100 \mathrm{~J} / \mathrm{mol}$, in agreement with Saldana et al. $(2012)^{3}$.

Figure 14 shows the distribution of the ratio of the latent heat stored by the grain boundaries $U_{d}$ to the total latent heat $U_{m}$. The value of $U_{m}$ is obtained as specified at the previous paragraph while, considering only the second term on the right-hand side of eq. (6), we have $U_{d}$. A maximum value of $83.34 \%$ is predicted within the regions of maximum grain refinement in the red contour. This intuitively suggests that grain refinement is a process that builds a significant amount of grain boundary surface, from which, the grain boundary energy $U_{d}$. The material in annealed conditions shows the minimum value of $0.92 \%$ in the blue contour, as in this condition, the latent heat of the material is stored primarily by the dislocation network.

\section{Discussion}

In the proposed model, the influence of the temperature, on grain refinement and on the evolution and mechanisms of energy storage, has not been investigated. The temperature is also expected to affect the critical grain size $d_{c}$, as relevant GBS has been observed for micrometric grain size in the form of high temperature creep (Langdon 1994). In this model, we adopted a constant average temperature that has a value sufficiently close to its room one $\mathrm{T}_{r}$ in order to allow us to neglect its influence on the studied phenomena. This hypothesis is supported by a negligible temperature rise observed in most of the experiments taken for comparison. Shankar et al. (2005) observed "negligible temperature rise" during their machining test, hypothesis confirmed by the study of Abolghasem et al. 2012, which measured a $\Delta \mathrm{T}$ that is below $50^{\circ} \mathrm{K}$, Farren and Taylor (1925) observed a temperature rise $\Delta \mathrm{T}$ that is around $3^{\circ} \mathrm{K}\left(0.01 \mathrm{~T}_{r}\right)$ and Rosenhein and Stott (1933) observed $\Delta \mathrm{T} \approx 20^{\circ} K\left(0.07 \mathrm{~T}_{r}\right)$. A more relevant temperature rise, in the range of $100^{\circ} \mathrm{K}\left(0.34 \mathrm{~T}_{r}\right)$, is observed in the results of Mason et al. (1994) and Hodowany et al. (2000). In this regard, the comparison made in Figure 6 has to be considered qualitative. Thermal fluctuation in adiabatic conditions is also supposed to activate recrystallization for

\footnotetext{
${ }^{3}$ Saldana et al. (2012) performed machining experiments on copper specimens, while the latent heat we calculated is related to an aluminum alloy. We expect to have a qualitative matching in this regard as the materials are different.
} 
temperatures above $0.4 \mathrm{~T}_{m}$ (Meyers et al. 2003). Considering a final temperature of $350^{\circ} \mathrm{K}$ (Abolghasem et al. 2012) and $0.4 \mathrm{~T}_{m}=373^{\circ} \mathrm{K}$ for aluminum, we consider this effect to be negligible for the purpose of this investigation.

The influence of strain rate has been considered indirectly in the proposed model. It is expected to affect the yield strength in shear at steady state $\tau_{c}$, as well as the critical amount of shear equivalent plastic strain $\gamma_{c}$ developed before $\tau_{y}$ reaches $\tau_{c}$ and CDRX begins. The value of $\tau_{c}$ has been obtained by the comparison with experimental measurement, on the Vicker hardness $H_{v}$, made by Shankar et al. (2005). In this way, the strain rate adopted in such experiment has been considered in the phenomena through calibration. The aforementioned authors did not directly measure the strain rate, however, an estimation has been done from the finite element simulation used to obtain Figures 9-13, and from empirical formulations, which gives a value of $\dot{\gamma} \approx 300 \mathrm{~s}^{-1}$ . In Figures 5-6, the comparison between theoretical and experimental results is made with different strain rates. This comparison is supported by the little influence of the strain rate on the evolution of the T\&Q coefficient observed by Hodowany et al. (2000). From this observation, we expect to obtain little influence from strain rate also on grain refinement, in virtue of the formulation obtained from the substitution of eq. (16) into eq. (5).

\section{Conclusions}

A description of the phenomenon of LHS has been provided in this paper. Its relationship with grain refinement due to CDRX has also been explained with the proposed thermodynamically consistent model. This model gives a phenomenological description of the microstructural evolution of polycrystalline materials, showing a final refined nanostructure obtainable with large plastic deformations. For metal alloys, this nanostructure is stable at room temperature and gives improved material properties that can be conserved at higher temperatures according to the design of the material. This stability is due to grain boundary pinning by second phase precipitates which, in this way, prevents the annealing phenomenon related to grain growth. Ultimately, this consideration underlines the importance of grain refinement in manufacture engineering and material science and stresses the importance of our study.

In the presented work, the temperature rise given by internal heat generation has been considered negligible. This hypothesis is supported by accordant observations found in the referred experimental work. This framework is extended from low to high strain rate compression and tension tests, and to low cutting speed machining. For different typologies of experiments, the temperature rise is expected to give relevant influence in the phenomena of study, thus it needs to be accounted.

If the temperature rise is neglected, a constant critical grain size $d_{c}$, for the inversion of the HallPetch effect, is considered for any amount of plastic strain. This grain size represents the asymptotic value for grain refinement, leading to the conclusion that a smaller grain size cannot be obtained with CDRX. As a consequence, no inverse Hall-Petch behavior is observed during grain refinement.

The evolution of the grain size, the latent heat and the T\&Q coefficient, for continued plastic strain, is predicted by the proposed model and this prediction has been validated by experiments. 
Acknowledgments - The authors would like to acknowledge support from U.S. DOE Grant DEFG03-99ER-45768 (via Third Wave Systems). The authors also thank Professor Srinivasan Chandrasekar (Purdue University), Professor Christopher Saldana (Georgia Tech), Professor David Hayhurst (University of Manchester), Professor Thresa Pollok and Professor Carlos Levi (UCSB) for useful discussions.

Disclaimer - This report was prepared as an account of work sponsored by an agency of the United States Government. Neither the United States Government nor any agency thereof, nor any of their employees, makes any warranty, express or implied, or assumes any legal liability or responsibility for the accuracy, completeness, or usefulness of any information, apparatus, product, or process disclosed, or represents that its use would not infringe privately owned rights. Reference herein to any specific commercial product, process, or service by trade name, trademark, manufacturer, or otherwise does not necessarily constitute or imply its endorsement, recommendation, or favoring by the United States Government or any agency thereof. The views and opinions of authors expressed herein do not necessarily state or reflect those of the United States Government or any agency thereof.

\section{References}

S. Abolghasem, S. Basu, S. Shekhar, J. Cai, M.R. Shankar (2012), "Mapping subgrain sizes resulting from severe simple shear deformation", Acta Mater. 60 (1) pp. 376-386.

E.C. Aifantis (1984), "On the microstructural origin of certain inelastic models", Trans. ASME J. Eng. Mater. Technol. 106 (4) pp. 326-330.

M. Bacca, D. Bigoni, F. Dal Corso, D. Veber (2013), "Second-gradient elastic properties from dilute two-phase Cauchy-elastic composites. Part I: Closed form expression for the effective higher-order constitutive tensor", Int. J. Solids Struct. 50 (24) pp. 4010-4019.

M. Bacca, D.R. Hayhurst, R.M. McMeeking (2015), "Continuous Dynamic Recrystallization during Severe Plastic Deformation", Mech. Mater. 90 pp. 148-156.

J.E. Bailey, P.B. Hirsch (1962), "The Recrystallization Process in Some Polycrystalline Metals", Proc. R. Soc. A 267 (1328) pp. 11-30.

V.L. Berdichevsky (2006), "On thermodynamics of crystal plasticity", Scripta Mater. 54 (5) pp. 711-716.

M. Bernacki, H. Resk, T. Coupez, R.E. Logé (2009), "Finite element model of primary recrystallization in polycrystalline aggregates using a level set framework", Model. Simul. Mater. Sci. Eng. 17 (6).

M.B. Bever, D.L. Holt, A.L. Titchener (1973), "The Stored Energy of Cold Work", Prog. Mater. Sci. 17 pp. 1-192.

A.A. Brown, D.J. Bammann (2012), "Validation of a model for static and dynamic recrystallization in metals", Int. J. Plasticity 32-33 pp. 17-35. 
F.R. Castro-Fernáandez, C.M. Sellars (1989), "Relationship between room-temperature proof stress, dislocation density and subgrain size", Philos. Mag. A 60 (4) pp. 487-506.

Y. Chang, D.M. Kochmann (2015), "A variational constitutive model for slip-twinning interactions in hep metals: Application to single- and polycrystalline magnesium", Int. J. Plasticity 73 pp. 39-61.

A. Chokshi, A. Rosen, J. Karch, H. Gleiter (1989), "On the validity of the Hall-Petch relationship in nanocrystalline materials", Scripta Metall. 23 pp. (10) 1679-1684.

W.S. Farren, G.I. Taylor (1925), "The Heat Developed during Plastic Extension of Metals", Proc. R. Soc. A 107 (743) pp. 422-451.

E.O. Hall (1951), "The deformation and ageing of mild steel", Proc. Phys. Soc. B 64 (9) pp. $742-$ 753.

H. Hallberg (2011), "Approaches to Modeling of Recrystallization", Metals 1 (1) pp. 16- 48.

J. Hodowany, G. Ravichandran, A.J. Rosakis, P. Rosakis (2000) "Partition of Plastic Work into Heat and Stored Energy in Metals", Exp. Mech. 40 (2) pp. 113-123.

H. Kanzaki (1951) "On the Recovery Process of Cold Worked Metals", J. Phys. Soc. Jpn. 6 (2) pp. 90-94.

U.F. Kocks (1976), "Laws for Work-Hardening and Low-Temperature Creep", J. Eng. Mater. Technol. 98 (1) pp. 76-85.

T.G. Langdon (1994), "A unified approach to grain boundary sliding in creep and superplasticity", Acta Metall. Mater. 42 (7) pp. 2437-2443.

K.C. Le, D.M. Kochmann (2009), "A simple model for dynamic recrystallization during severe plastic deformation", Arch. Appl. Mech. 79 (6-7) pp. 579-586.

M.J. Luton, C.M. Sellars (1969), "Dynamic recrystallization in nickel and nickel-iron alloys during high temperature deformation", Acta Metall. 17 (8) pp. 1033-1043.

J.J. Mason, A.J. Rosakis, G. Ravichandran (1994) "On the strain and strain rate dependence of the fraction of plastic work converted to heat: an experimental study using high speed infrared detectors and the Kolsky bar", Mech. Mater. 17 (2-3) pp. 135-145.

K. Maung, J.C. Earthman, F.A. Mohamed (2012), "Inverse Hall-Petch behavior in diamantane stabilized bulk nanocrystalline aluminum", Acta Mater. 60 (16) pp. 5850-5857.

M.A. Meyers, Y.B. Xu, Q. Xue, M.T. Pérez-Prado, T.R. McNelley (2003), "Microstructural evolution in adiabatic shear localization in stainless steel", Acta Mater. 51 (5) pp. 1307-1325. 
M. Militzer (2011), "Phase field modeling of microstructure evolution in steels", Curr. Opin. Solid State Mater. Sci. 15 (3) pp. 106-115.

R.D. Mindlin (1964), "Micro-structure in linear elasticity", Arch. Ration. Mech. Anal. 16 (1) pp. $51-78$.

N. Moelans, A. Godfrey, Y. Zhang, D.J. Jensen (2013), "Phase-field simulation study of the migration of recrystallization boundaries", Phys. Rev. B 88 (5) 054103.

G. Nurislamova, X. Sauvage, M. Murashkin, R. Islamgaliev, R. Valiev (2008), "Nanostructure and related mechanical properties of an $\mathrm{Al}-\mathrm{Mg}-\mathrm{Si}$ alloy processed by severe plastic deformation", Philos. Mag. Lett. 88 (6) pp. 459-466.

N.J. Petch (1953), "The cleavage strength of polycrystals", J. Iron Steel Inst. 174 pp. 25-28.

G.Z. Quan, Y. Shi, Y.X. Wanga, B.S. Kang, T.W. Kub, W.J. Song (2011), "Constitutive modeling for the dynamic recrystallization evolution of AZ80 magnesium alloy based on stressstrain data", Mat. Sci. Eng. A 528 (28) pp. 8051-8059.

R. Raj, M.F. Ashby (1971), "On Grain Boundary Sliding and Diffusional Creep", Metall. Trans. 2 (4) pp. 1113-1127.

W. A. Rachinger (1952-53), "Relative grain translations in the plastic flow of aluminium", $J$. Inst. Metals 81 pp. 33-41.

W.T. Read, W. Shockley (1950), "Dislocation Models of Crystal Grain Boundaries", Phys. Rev. 78 pp. $275-289$.

W. Roberts, B. Ahlblom, (1978), "A nucleation criterion for dynamic recrystallization during hot working", Acta Metall. 26 (5) pp. 801-813.

W. Rosenhain, V. H. Stott, (1933), "The Energy Absorbed in the Cold Working of Metals", Proc. R. Soc. A 140 (840) pp. 9-25.

J. Rösler, H. Harders, M. Bäker (2007), "Mechanical Behaviour of Engineering Materials" Springer-Verlag Berlin.

C. Saldana, A.H. King, S. Chandrasekar (2012), "Thermal stability and strength of deformation microstructures in pure copper", Acta Mater. 60 (10) pp. 4107-4116.

Z.W. Shan, E.A. Stach, J.M.K. Wiezorek, J.A. Knapp, D.M. Follstaedt, S.X. Mao (2004), "Grain Boundary-Mediated Plasticity in Nanocrystalline Nickel", Science 305 (5684) pp. 654-657.

M.R. Shankar, S. Chandrasekar, A.H. King, W.D. Compton (2005), "Microstructure and stability of nanocrystalline aluminum 6061 created by large strain machining", Acta Mater. 53 (18) pp. 4781-4793. 
S. Sreekala, M. Haataja (2007), "Recrystallization kinetics: A coupled coarse-grained dislocation density and phase-field approach", Phys. Rev. B 76 (9) 094109.

Y. Suwa, Y. Saito, H. Onodera (2008), "Phase-field simulation of recrystallization based on the unified subgrain growth theory", Comput. Mater. Sci. 44 (2) pp. 286-295.

G.I. Taylor (1934), "The mechanism of plastic deformation of crystals", Proc. R. Soc. A 145 (855) pp. 362-387.

G.I. Taylor, H. Quinney (1934), "The latent energy remaining in a metal after cold working", Proc. R. Soc. A 143 (849) pp. 307-326.

E. Voce (1948), "The Relationship between Stress and Strain for Homogeneous Deformation", $J$. Inst. Metals 74 pp. 537-562. 


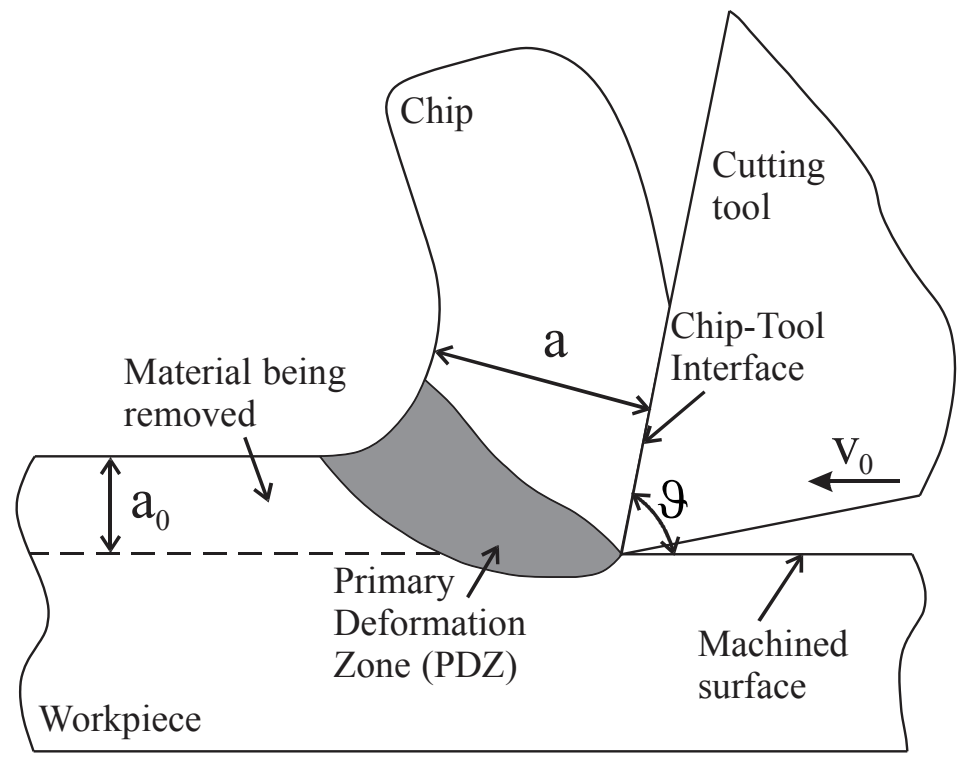

Figure 1: Schematic representation of the machining experiment used for comparison of the proposed model. In this picture, the key parameters for machining are shown and their value for the experiment of Shankar et al. (2005) are reported in Table 1. 


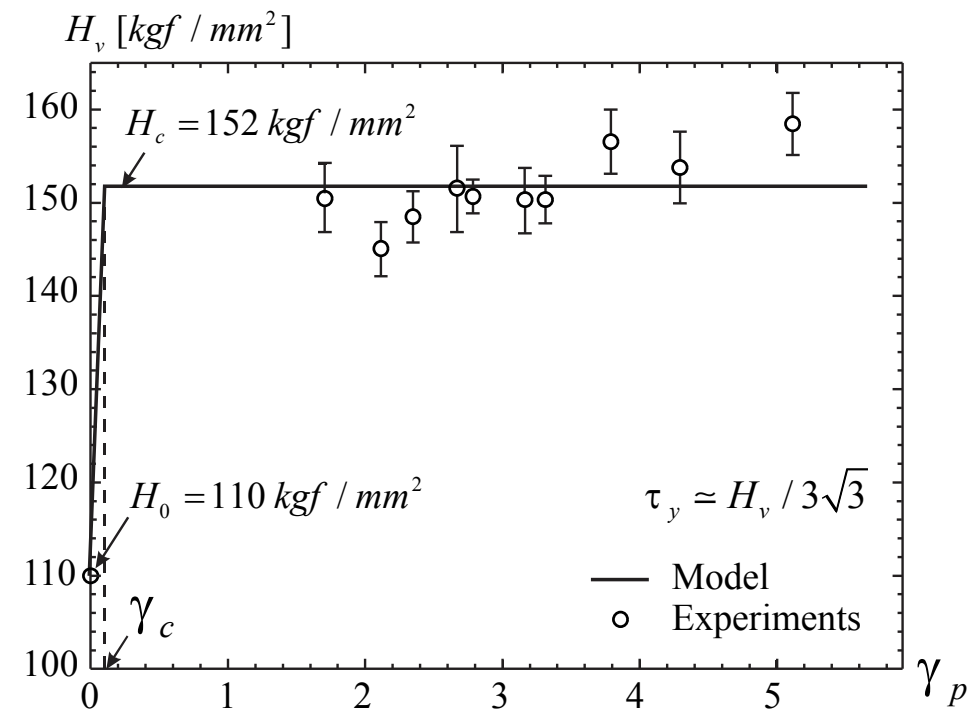

Figure 2: For experiments on machining, variation of the Vicker hardness $H_{v}$ within the chip for various values of the shear equivalent plastic strain $\gamma_{p}$. The solid line indicates the model prediction while the experimental values are indicated with open circles and relative error bars. $\gamma_{c}$ is the critical strain at which recrystallization begins. The material used in the experiments is the peak-aged Aluminum 6061, $H_{0}$ is the hardness prior to machining and $H_{c}$ is the hardness of all the machined samples for which $\gamma_{p}>\gamma_{c}$. The Vickers indentation load is $50 \mathrm{~g}$ (Shankar et al., 2005). 


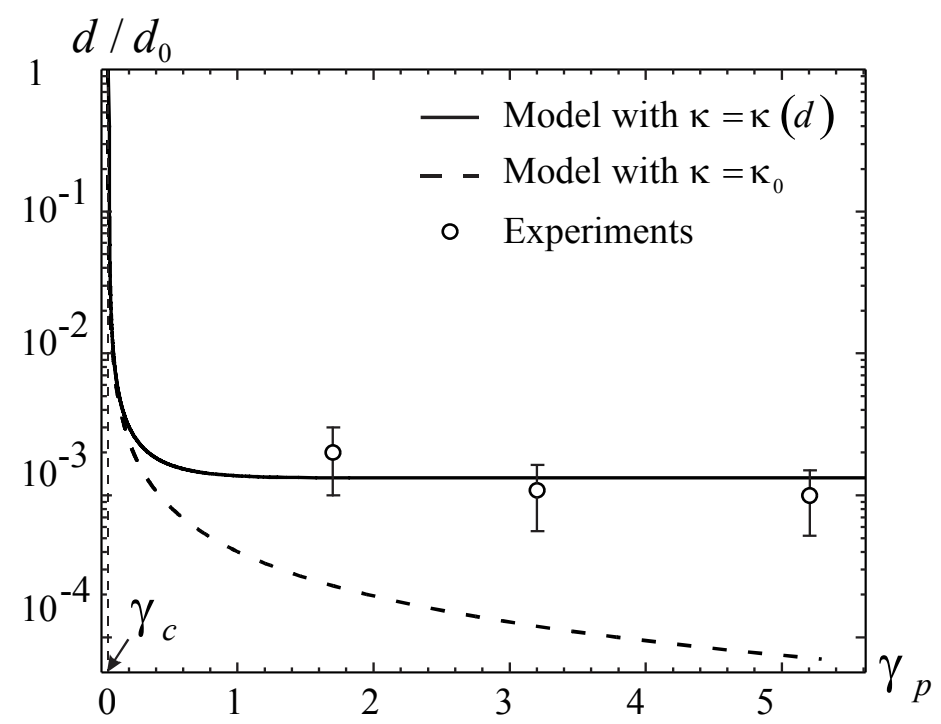

Figure 3: Evolution of the grain size $d$ as a function of the shear equivalent plastic strain $\gamma_{p}$ relative to the experiments in Figure 2. In the ordinate, we have the ratio $d / d_{0}$ in a logarithmic scale, where $d_{0}$ is the grain size at the annealed state. The solid line indicates the prediction made with the proposed model, with a microstructure dependent latent heat capacity $\kappa=\kappa(d)$, the dashed line indicates the prediction made with our previous model (Bacca et al. 2015) which assumes constant latent heat capacity $\kappa=\kappa_{0}$ (with $\kappa_{0}$ the value associated with zero plastic strain), and the open circles with error bars indicate the experimental results on machining (Shankar et al., 2005). 


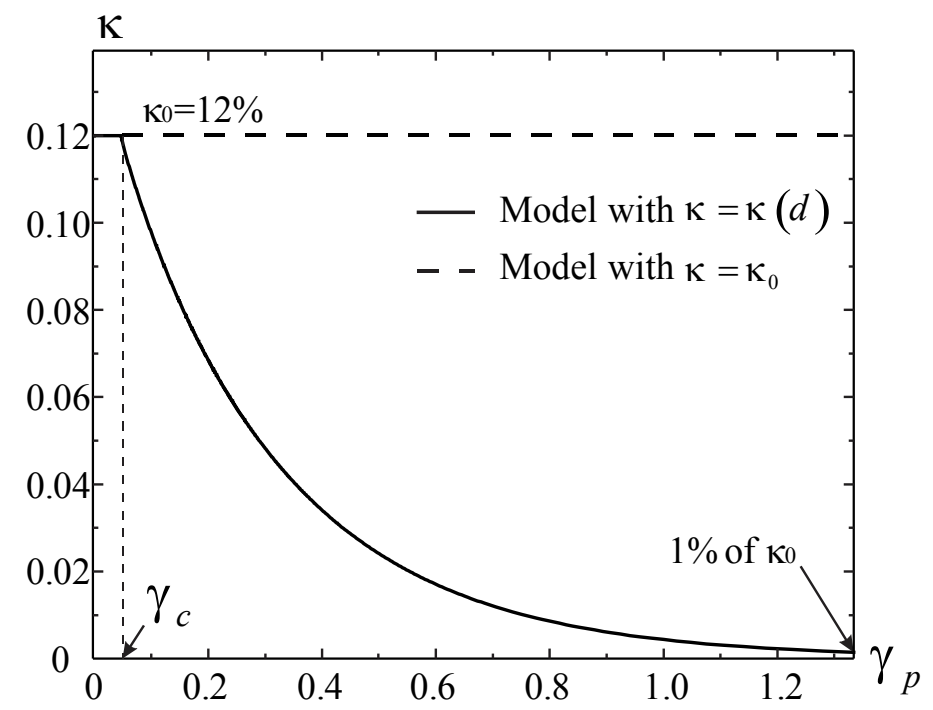

Figure 4: Evolution of the latent heat capacity $\kappa$ as a function of the shear equivalent plastic strain $\gamma_{p}$ relative to the change in microstructure shown in Figures 3 . The solid line indicates the prediction made with the proposed model, with a microstructure dependent latent heat capacity $\kappa=\kappa(d)$, while the dashed line indicates the prediction made with our previous model (Bacca et al. 2015) which assumes $\kappa=\kappa_{0}$. 


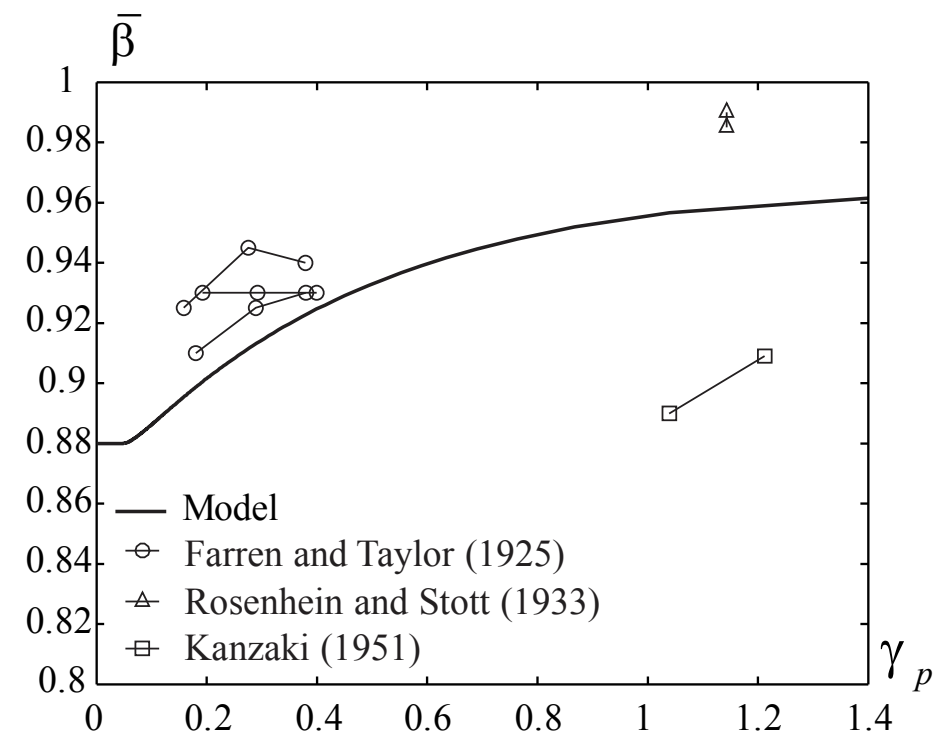

Figure 5: Evolution of the mean Taylor \& Quinney coefficient $\bar{\beta}$ as a function of the shear equivalent plastic strain $\gamma_{p}$ relative to the change in microstructure shown in Figures 3 . The solid line indicates the prediction made with the proposed model while the open polygons indicate the experimental values taken from Farren and Taylor (1925), Rosenhein and Stott (1933) and Kanzaki (1951). 


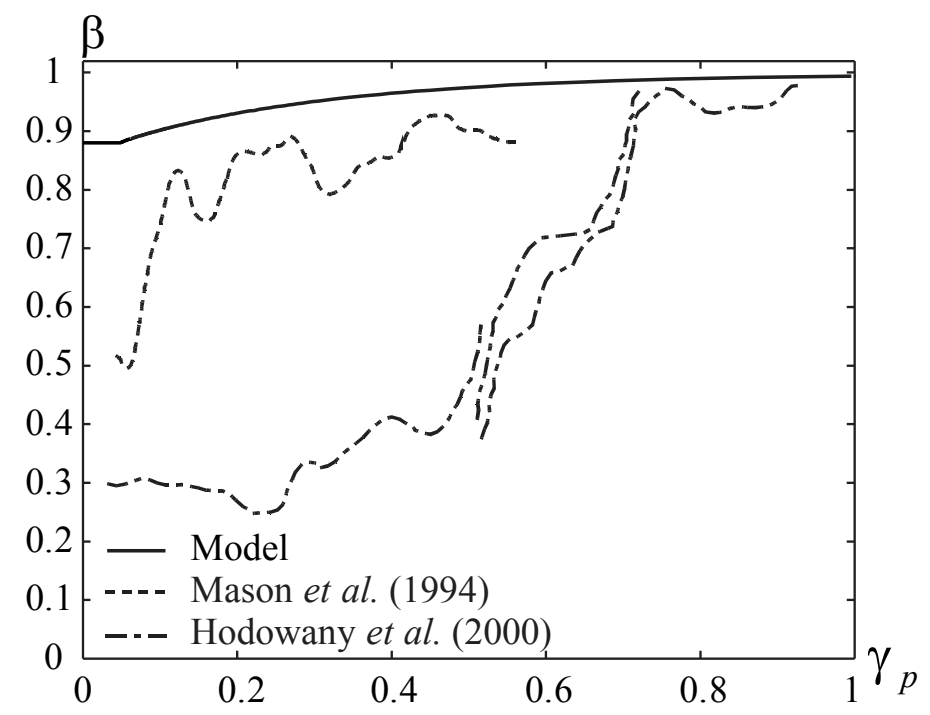

Figure 6: Evolution of the Taylor \& Quinney coefficient $\beta$ as a function of the shear equivalent plastic strain $\gamma_{p}$ relative to the change in microstructure shown in Figures 3. The solid line indicates the prediction made with the proposed model while the dashed lines indicate the experimental curves taken from Mason et al. (1994) and Hodowany et al. (2000). 


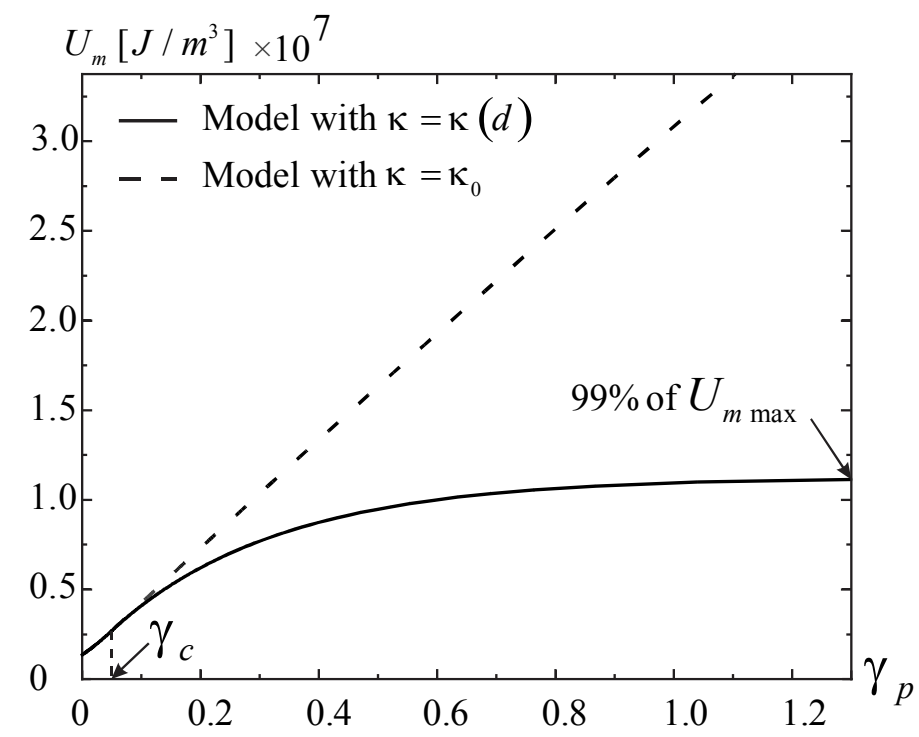

Figure 7: Evolution of the latent heat $U_{m}$ as a function of the shear equivalent plastic strain $\gamma_{p}$ relative to the change in microstructure shown in Figures 3. The solid line indicates the prediction made with the proposed model, with a microstructure dependent latent heat capacity $\kappa=\kappa(d)$, while the dashed line indicates the prediction made with our previous model (Bacca et al. 2015) which assumes $\kappa=\kappa_{0}$. 


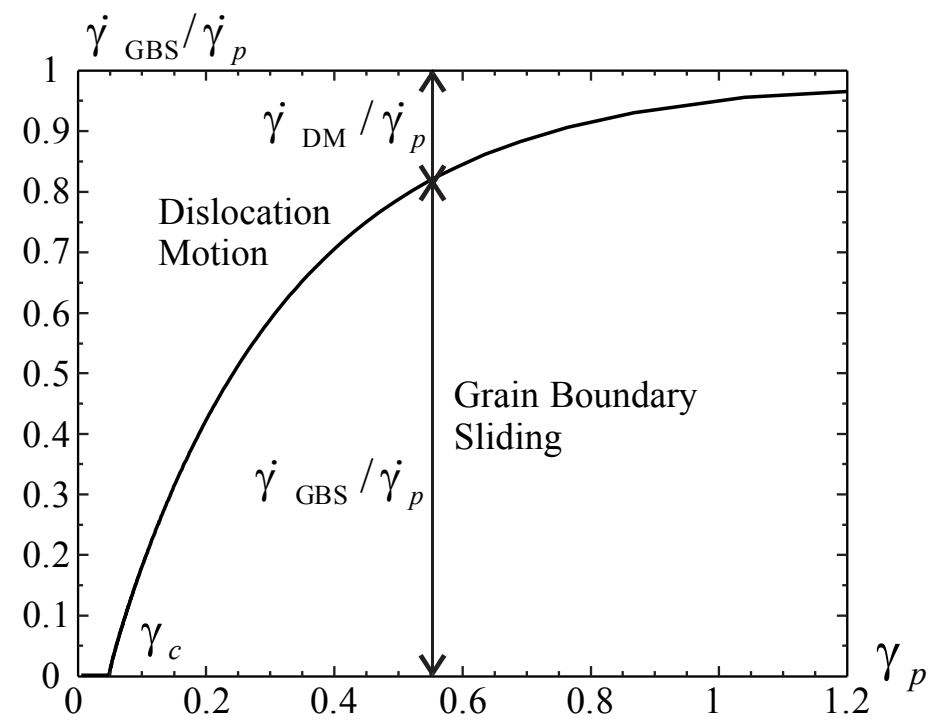

Figure 8: Evolution of the ratio of the strain rate $\dot{\gamma}$ GBS created by GBS to the total plastic strain rate $\dot{\gamma}_{p}$ as a function of the shear equivalent plastic strain $\gamma_{p}$ relative to the change in microstructure shown in Figures 3. 


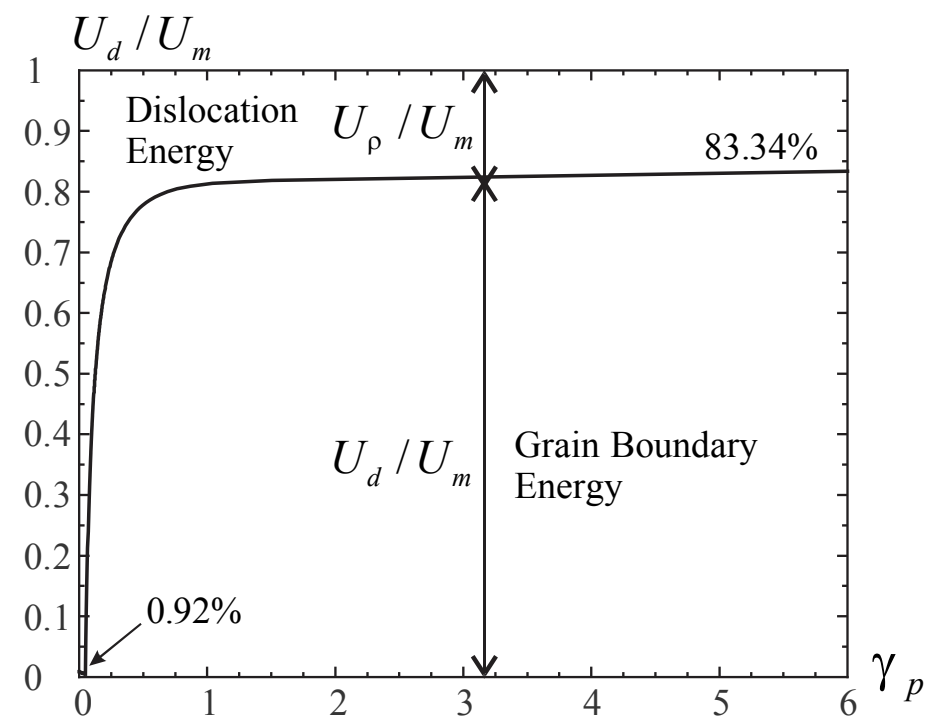

Figure 9: Evolution of the ratio of the energy stored by the grain boundaries $U_{d}$ to the total latent heat $U_{m}$ as a function of the shear equivalent plastic strain $\gamma_{p}$ relative to the change in microstructure shown in Figures 3. 


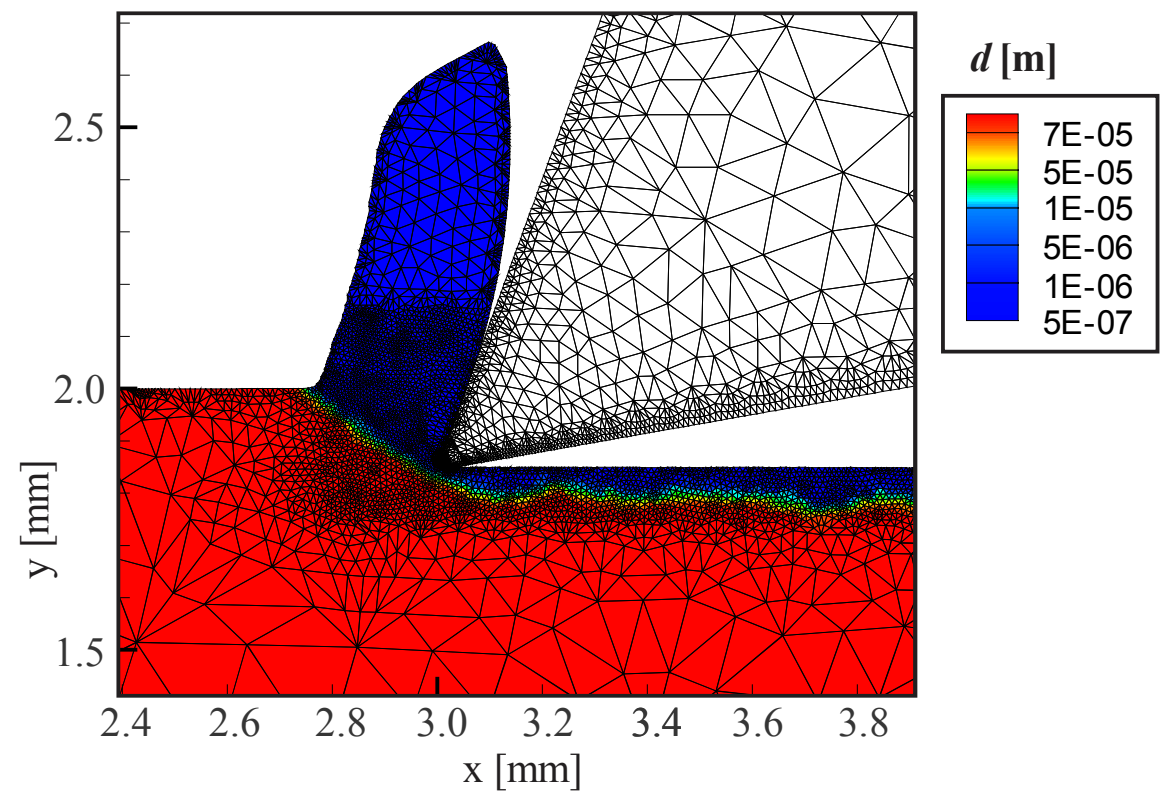

Figure 10: Contour plot of the average grain size $d$ for machining predicted with the finite element software Advant Edge (Third Wave Systems) [http://www. thirdwavesys.com/]. The friction coefficient between the cutting tool and the chip is 0.5 , the cutting speed is $20 \mathrm{~mm} / \mathrm{s}$, the rake angle is $20^{\circ}$, the feed $\left(a_{0}\right)$ is $0.15 \mathrm{~mm}$, and the material of the specimen is Al6061-T6. The smallest value is $100 \mathrm{~nm}$ in the blue contour while the largest value is $75 \mu \mathrm{m}$ in the red contour. 


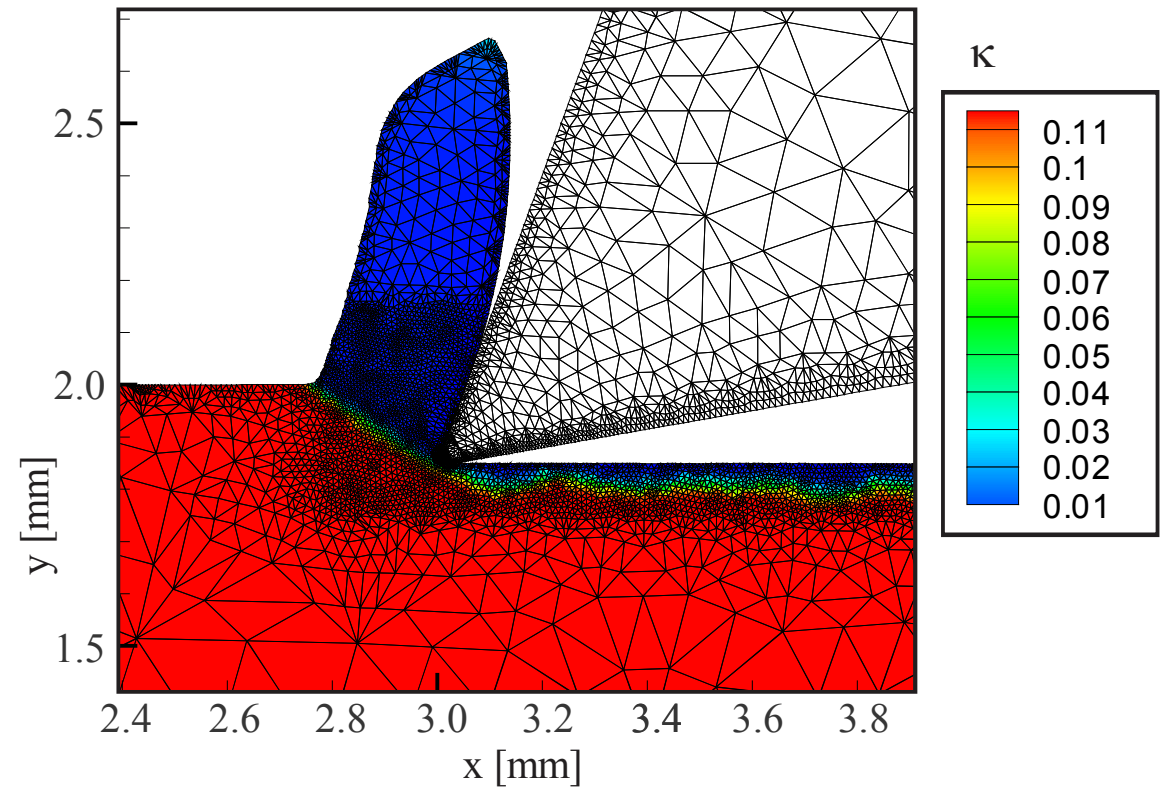

Figure 11: Contour plot of the latent heat capacity $\kappa$ for machining. See Figure 9 for details on the simulation. The smallest value is 0 in the blue contour while the largest value is 0.12 in the red contour. 


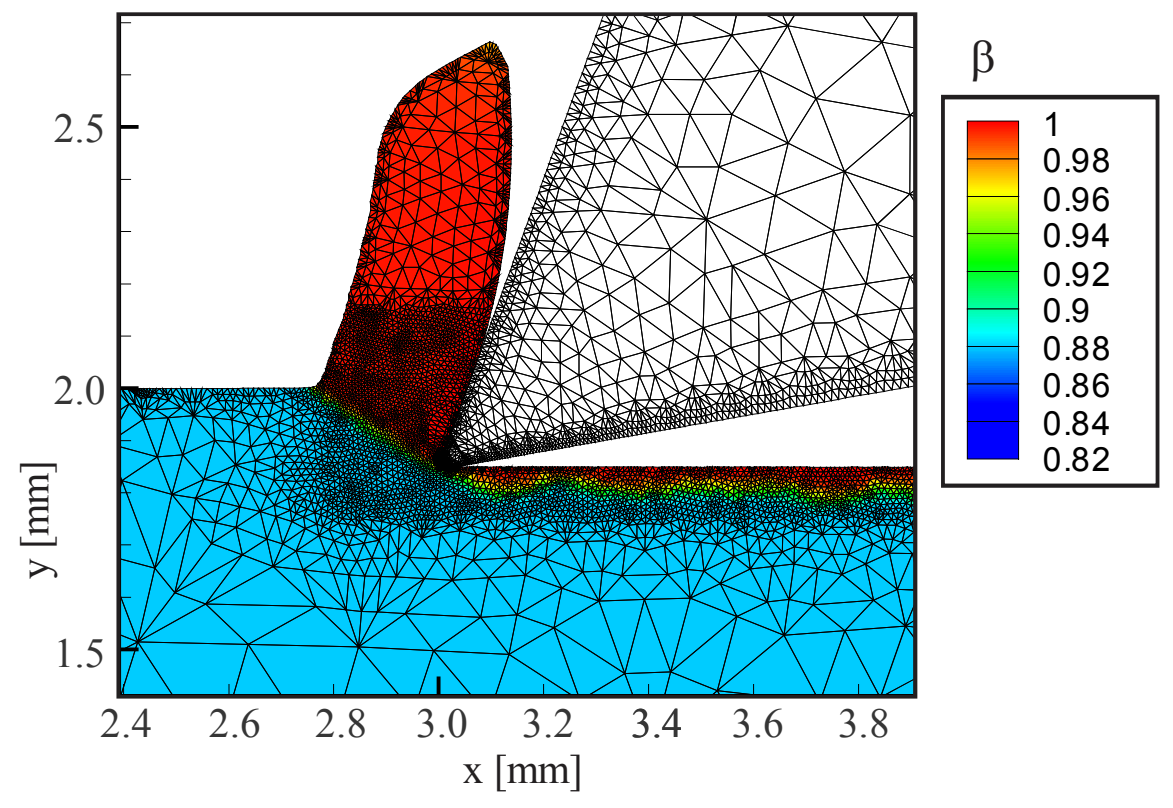

Figure 12: Contour plot of the Taylor and Quinney coefficient $\beta$ for machining. See Figure 9 for details on the simulation. The largest value is 1 in the red contour while the smallest value is 0.88 in the cyan contour. 


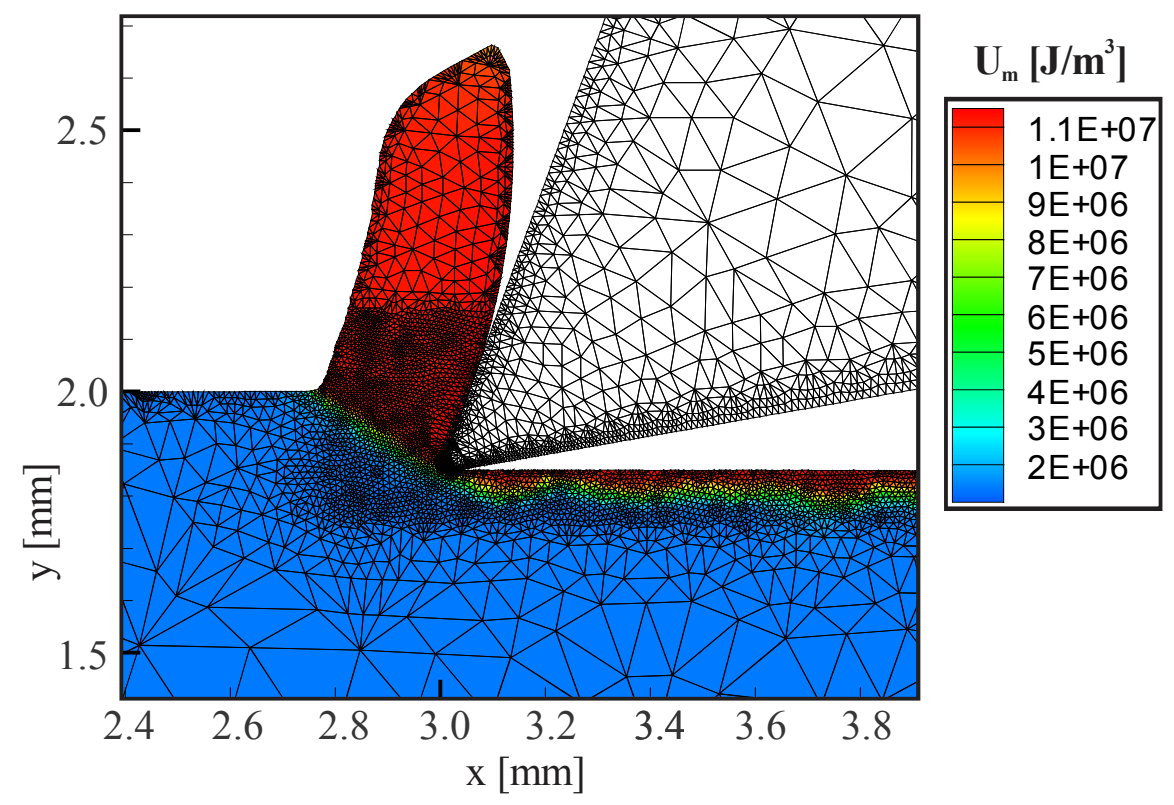

Figure 13: Contour plot of the Latent Heat $U_{m}$ for machining. See Figure 9 for details on the simulation. The largest value is $1.1410^{7}$ in the red contour while the smallest value is $1.3610^{6} \mathrm{~J} / \mathrm{m}^{3}$ in the blue contour. 


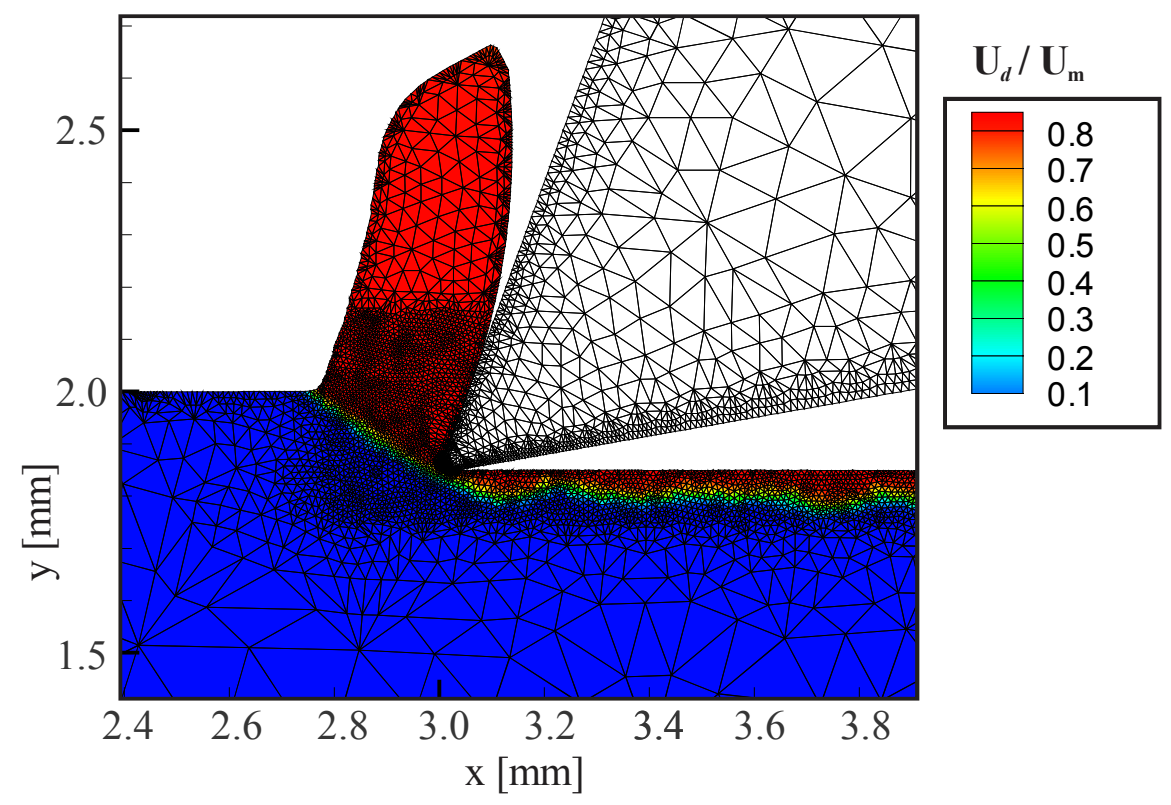

Figure 14: Contour plot of the ratio of the latent heat stored by the grain boundaries $U_{d}$ to the total Latent Heat $U_{m}$ for machining. See Figure 9 for details on the simulation. The largest value is $83.34 \%$ in the red contour while the smallest value is $0.92 \%$ in the blue contour. 